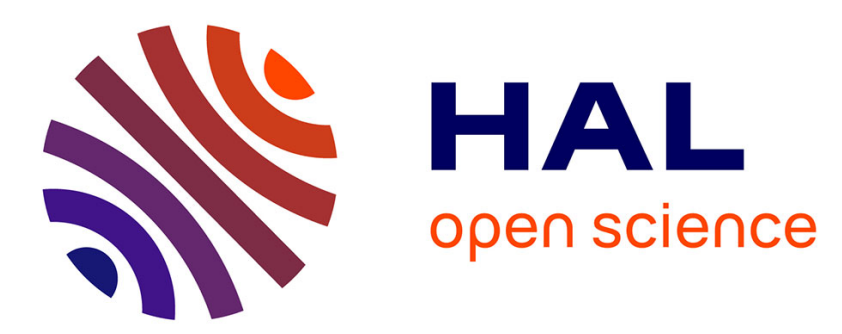

\title{
Comparison of Geant4-DNA simulation of S-values with other Monte Carlo codes
}

\author{
T. André, Filippo Morini, M. Karamitros, R. Delorme, C. Le Loirec, L. \\ Campos, C. Champion, J.-E. Groetz, M. Fromm, M.-C. Bordage, et al.
}

\section{- To cite this version:}

T. André, Filippo Morini, M. Karamitros, R. Delorme, C. Le Loirec, et al.. Comparison of Geant4DNA simulation of S-values with other Monte Carlo codes. Nuclear Instruments and Methods in Physics Research Section B: Beam Interactions with Materials and Atoms, 2014, 319, pp.87-94. 10.1016/j.nimb.2013.11.005 . in2p3-00914193

\section{HAL Id: in2p3-00914193 https://hal.in2p3.fr/in2p3-00914193}

Submitted on 11 Jul 2019

HAL is a multi-disciplinary open access archive for the deposit and dissemination of scientific research documents, whether they are published or not. The documents may come from teaching and research institutions in France or abroad, or from public or private research centers.
L'archive ouverte pluridisciplinaire HAL, est destinée au dépôt et à la diffusion de documents scientifiques de niveau recherche, publiés ou non, émanant des établissements d'enseignement et de recherche français ou étrangers, des laboratoires publics ou privés. 


\section{Comparison of Geant4-DNA simulation of S-values with other Monte Carlo codes}

T. Andréab1, F. Morinic, M. Karamitros ${ }^{\mathrm{abd} 1}$, R. Delorme ${ }^{\mathrm{ef}}$, C. Le Loirec ${ }^{f}$, L. Campos ${ }^{\mathrm{g}}, \mathrm{C}$. Champion $^{\text {ab1 }}$, J.-E. Groetz ${ }^{h}$, M. Fromm ${ }^{\text {h }}$, M.-C. Bordage ${ }^{i 1}$, Y. Perrot ${ }^{1}$, Ph. Barberet ${ }^{\text {ab1 }}$, M.A. Bernal $^{k 1}$, J.M.C. Brown ${ }^{11}$, M.S. Deleuze ${ }^{c}$, Z. Francis ${ }^{\mathrm{mn} 1}$, V. Ivanchenko ${ }^{\mathrm{op} 1}$, B. Mascialino ${ }^{\mathrm{q} 1}$, C. Zacharatou $^{r 1}$, M. Bardiès ${ }^{\mathrm{s} 1}$, S. Incertiab

a Université Bordeaux 1, CENBG, UMR 5797, F-33170 Gradignan, France

${ }^{\mathrm{b}}$ CNRS, IN2P3, CENBG, UMR 5797, F-33170 Gradignan, France

${ }^{c}$ Research Group of Theoretical Chemistry and Molecular Modelling, Hasselt University, Agoralaan Gebouw D, B-3590 Diepenbeek, Belgium

${ }^{\mathrm{d}}$ CNRS, INCIA, UMR 5287, F-33400 Talence, France

e LPSC, Université Joseph Fourier Grenoble 1, CNRS/IN2P3, Grenoble INP, 38026 Grenoble, France

${ }^{f}$ CEA, LIST, F-91191 Gif-sur-Yvette, France

${ }^{9}$ Departamento de Física, Universidade Federal de Sergipe, São Cristóvão, Brazil

h Université de Franche-Comté, Laboratoire Chrono-Environnement, UMR CNRS 6249, Besançon, France

'Laboratoire Plasmas et Conversion d'Énergie, UMR 5213 CNRS-INPT-UPS, Université Paul

Sabatier, Toulouse, France

'Laboratoire de Physique Corpusculaire, UMR 6533, Aubière, France

${ }^{k}$ Instituto de Física Gleb Wataghin, Universidade Estadual de Campinas, SP, Brazil

'School of Physics, Monash University, Melbourne, Australia

m Université Saint Joseph, Science Faculty, Department of Physics, Beirut, Lebanon

${ }^{n}$ The Open University, Faculty of Science, Department of Physical Sciences, Walton Hall, MK7

6AA Milton Keynes, United Kingdom

${ }^{\circ}$ Ecoanalytica, 119899 Moscow, Russia

${ }^{\mathrm{P}}$ Geant4 Associates International Ltd., United Kingdom

q Thermo Fisher Scientific, Rapsgatan 7, 75137 Uppsala, Sweden

'Institut Bergonié, Bordeaux, France

${ }^{\mathrm{s}}$ CRCT, UMR 1037 INSERM, Université Paul Sabatier, Toulouse, France

\section{Abstract}

Monte Carlo simulations of $S$-values have been carried out with the Geant4-DNA extension of the Geant4 toolkit. The $S$-values have been simulated for monoenergetic electrons with energies ranging from $0.1 \mathrm{keV}$ up to $20 \mathrm{keV}$, in liquid water spheres (for four radii, chosen between $10 \mathrm{~nm}$ and $1 \mu \mathrm{m})$, and for electrons emitted by five isotopes of iodine $(131,132,133$, 134 and 135), in liquid water spheres of varying radius (from $15 \mu \mathrm{m}$ up to $250 \mu \mathrm{m}$ ). The results have been compared to those obtained from other Monte Carlo codes and from other published data. The use of the Kolmogorov-Smirnov test has allowed confirming the statistical compatibility of all simulation results.

\section{Keywords}

Geant4-DNA, Monte Carlo, Liquid water, S-values 


\section{Introduction}

Radiation therapy is a cancer treatment technique that consists in irradiating tumor tissues in order to kill malignant cells by mainly damaging nuclear desoxyribonucleic acid (DNA) and sparing as much as possible surrounding healthy tissues. Two types of treatments can be then distinguished, namely, external and internal radiation therapy. While the former consists in irradiating patients with external beams of photons, electrons, protons or ions, the latter implies the use of a radioactive source in the close vicinity of tumor cells. In this context, two techniques should be distinguished: brachytherapy and targeted internal radiation therapy. In brachytherapy [1], radiation sources (usually enclosed within non-radioactive capsules) are implanted close to the tumor in the form of seeds, wires, needles, or plaques. Targeted internal radiation therapy [2], [3] is helpful in the case of tumors that are small and disseminated in the organism. It is based on the particular affinity between the radioactive substance and the tumor cells, mostly by means of a vector. The radioactive agent is fixed on this vector, which preferentially attaches to the tumor cells. The radioactive substance can be injected intravenously. The choice of the radioisotope is essential [4] and must be adapted to the pathology. For example, Auger-emitting radionuclides have a strong potential for therapy [5] since they can deliver low energy electrons with very short ranges, close to the cancer metastases, preserving at maximum surrounding tissues. This treatment modality is original since it combines a systemic approach (like chemotherapy) with a mode of action based on tissue irradiation (like radiation therapy). Whether they are $\alpha$ particles, $\beta$ particles, Auger electrons or electrons from internal conversion, several criteria for selecting the radioisotope and the vector have to be considered [2], [4]:

- the emitted particles energies, which determine their range, i.e. the average distance they travel through biological matter,

- the half-life period of the isotope,

- the fixation of the isotope on the vector, which needs to be efficient and stable, in order to avoid any break and prevent irradiation of healthy surrounding tissues,

- the conditions of use in a routine treatment: the isotope must be abundant in nature, and its production can't be too costly.

The selective character of vectorized internal radiation therapy for specific cancer cells can be obtained through various vectors. In the simplest case, the radioisotope is injected and acts directly on the cells [2] because of the affinity between the obtained radioisotope-vector molecule and a particular cell line. This is the case with ${ }^{131}$ I for differentiated thyroid cancer. Monoclonal antibodies are an important class of vectors. They are immune system proteins that are specific to an antigen associated with a particular type of cancer. A particular technique involving monoclonal antibodies is the pre-targeting, or Affinity Enhancement System [4]. This method implies two successive injections, namely, a first one for the antibodies and a second one for the radioactive agent. The advantages are an efficient and long-term fixation on tumors, with a noticeable decrease of irradiation to healthy tissues. Besides, peptide radiolabelling [2], [6], [7], [8] is another alternative. These molecules, also called radio-ligands, are specific to a given receptor, overexpressed in case of cancer pathology.

Internal dosimetry of radionuclides is based on the framework developed by the committee on Medical Internal Radiation Dose (MIRD) [9], [10], [11]. This committee aims at providing standard methods and mathematical models for assessing internal radiation doses from administered radiopharmaceuticals. The MIRD approach simplifies the problem of assessing dose for many radionuclides in the human organs, tissues, and fluid compartments. In internal radiotherapy, the dose absorbed by a specific region of the body is expressed as:

$$
\bar{D}\left(r_{T} \leftarrow r_{S}\right)=\widetilde{A_{r_{S}}} S\left(r_{T} \leftarrow r_{S}\right)
$$


where $\mathrm{r}_{\mathrm{S}}$ is the source region, $\mathrm{r}_{\mathrm{T}}$ the target region, $\bar{D}\left(r_{T} \leftarrow r_{S}\right)$ the average dose absorbed by the target region, $\widetilde{A_{r_{S}}}$ the cumulated activity of the source region, and $S\left(r_{T} \leftarrow r_{S}\right)$ the $S$-value, on which we focus the current study. These are useful quantities related to the deposited energy at the level of the organ or the cell.

This quantity can be calculated notably by Monte Carlo techniques, which are used in a large variety of application domains. These methods, based on probability function sampling by use of random (or pseudo-random) numbers, enable to solve various problems, particularly those where stochastic processes are involved. Compared to the analytical ones, Monte Carlo methods provide more accurate physical models, finer geometrical descriptions (e.g. allowing inhomogeneities to be taken into account). In case of radiation therapy and more generally for the description of particle-matter interactions, it is thus possible to carry out detailed simulation of particle transport. The general scheme adopted for a simulation of a physical interaction follows a few steps in order to determine:

- the distance to the next physical interaction (according to the particle type and the medium),

- the material that interacts with the particle (according to the composition of the medium),

- the physical interaction type,

- the kinematics of the interaction, including the energy lost by the incident particle (locally deposited and/or transferred to the secondary particles potentially generated), the scattering and ejection directions.

These quantities are deduced from total and differential cross sections, the latter being calculated for each type of interaction by means of theoretical and/or semi-empirical models [12], [13], [14], [15], or by use of interpolation procedures from pre-calculated data tables. This sequence is repeated for each particle (primary as well as secondary) until it is locally absorbed, i.e. its energy becomes lower than a selected energy threshold, or exits from the region of interest.

Another solution adopted by some codes is to fix a "production threshold", in terms of energy or distance: the secondary particles are not explicitly simulated if their energy is not high enough to travel this distance in a given material.

The current work aims to compare the accuracy of the "Geant4-DNA" very low energy extension of the general-purpose Geant4 Monte Carlo simulation toolkit to a large set of Monte Carlo codes for the calculation of $S$-values. In a first stage, the accuracy of the codes will be compared for incident monoenergetic electron sources in nanometer size spherical geometries. Then, in a second stage, five isotopes of iodine will be simulated, as previously done in [16]. Furthermore, a simplified geometrical model of the colloid and the follicular cells of the thyroid will be used in both cases.

\section{Materials and methods}

\subsection{Monte Carlo codes}

Several Monte Carlo codes are currently available in the literature for simulating the radio induced interactions in biological matter. Among them, let us mention the Geant4-DNA extension of the Geant4 toolkit, CPA100, EGSnrc, EPOTRAN (with it's extension CELLDOSE), MCNP and PENELOPE. The accuracy of these codes has been compared in this work.

- The recent Geant4-DNA very low energy extension [15], [17], [18] is included in the generalpurpose Geant4 simulation toolkit [19], [20], a set of libraries written in C++, which enables to 
simulate particle transport and interactions in matter in a large variety of applications fields in high energy physics, space science and medical physics. Geant4-DNA allows the simulation of the step-by-step transport of particles in liquid water, including interactions of electrons, protons, hydrogen atoms and helium ions as well as a few ions $\left(\mathrm{C}^{6+}, \mathrm{N}^{7+}, \mathrm{O}^{8+}\right.$ and $\left.\mathrm{Fe}^{26+}\right)$. The covered energy ranges vary between $7.4 \mathrm{eV}$ and $1 \mathrm{MeV}$ for electrons, between $100 \mathrm{eV}$ and $100 \mathrm{MeV}$ for protons and hydrogen, and between $1 \mathrm{keV}$ and $400 \mathrm{MeV}$ for helium and its charged states. Geant4-DNA is mainly focused on the simulation of biological damages at the DNA scale;

- CPA100 [21] is a specific-purpose code, which simulates step-by-step the transport of electrons and photons in liquid water, for energies between $10 \mathrm{eV}$ and $200 \mathrm{keV}$. The simulation of physico-chemical and chemical stages (water radiolysis and damage of DNA) is also possible;

- EGSnrc [22] is another general-purpose code, enabling the simulation of electron and photon transport from a few keV up to $100 \mathrm{GeV}$; it is today considered as the "gold standard" for medical physics simulations;

- EPOTRAN [23] and more specifically CELLDOSE [16] is a specific-purpose simulation code for the step-by-step modelling of electron and positron transport, in liquid water and in gaseous state. The energy of the primary particles can vary between $7.4 \mathrm{eV}$ and $1 \mathrm{MeV}$;

- MCNP [24] is a general-purpose code for the transport of electrons, photons and neutrons. In the case of electrons, a condensed algorithm is used. Electrons can be transported from $1 \mathrm{keV}$ to $1 \mathrm{GeV}$.

- PENELOPE [25] is a general-purpose code enabling the simulation of electron, positron and photon transport. The available cross sections cover a large set of elements $(Z=1-99)$ and materials, for an energy range from $50 \mathrm{eV}$ up to $1 \mathrm{GeV}$;

A summary of energy ranges for the simulation of electron transport covered by these codes is presented in Table 1. All these codes are used in the context of medical physics, thus it is interesting to investigate their respective accuracy, in particular for targeted radiotherapy. Calculations of $S$-values (for ${ }^{131}$ I beta spectrum, in water spheres with radii between $150 \mu \mathrm{m}$ and $6 \mathrm{~mm}$ ) have been already described in the literature, e.g. with Geant4 [26], ETRAN [27] and PARTRAC [28], where it was shown that Geant4 was in good agreement with the other codes (it presented an underestimation of less than 5\%). The CELLDOSE code [16] based on the EPOTRAN code - has also been compared to ETRAN and PARTRAC simulations, and with results based on analytical methods [29], [30] (for ${ }^{131}$ I spectrum, in water spheres with radii between $50 \mathrm{~nm}$ and $1500 \mu \mathrm{m}$ ), and it was shown that the obtained data were in good agreement while the analytical values were a little lower (less than 10\%) and those from ETRAN and PARTRAC slightly higher (less than 10\%). In this work, we focus exclusively on the accuracy of the Geant4-DNA extension. 
Table 1. Summary of energy ranges for the simulation of electron transport covered by the various codes used in this study. Refer to text for further description of codes.

\begin{tabular}{|l|c|c|}
\hline Code & Minimum energy & Maximum energy \\
\hline Geant4-DNA & $7.4 \mathrm{eV}$ & $1 \mathrm{MeV}$ \\
\hline CPA100 & $10 \mathrm{eV}$ & $200 \mathrm{keV}$ \\
\hline EGSnrc & $\sim \mathrm{keV}$ & $100 \mathrm{GeV}$ \\
\hline CELLDOSE & $7.4 \mathrm{eV}$ & $1 \mathrm{MeV}$ \\
\hline MCNP & $1 \mathrm{keV}$ & $1 \mathrm{GeV}$ \\
\hline PENELOPE & $50 \mathrm{eV}$ & $1 \mathrm{GeV}$ \\
\hline
\end{tabular}

\subsection{A dedicated Geant4-DNA application}

In the current work, a dedicated application code called "svalue" has been developed for Geant4-DNA, version 9.6.p01 (February 2013). In a first stage, the Geant4-DNA simulations were carried out with monoenergetic electrons from $100 \mathrm{eV}$ to $20 \mathrm{keV}$. The emission is isotropic, and homogeneously distributed within nanometer size spheres of liquid water, with radii 10, 50, 100 and $1000 \mathrm{~nm}$, as found in the literature [31]. The absorbed dose is scored in the sphere.

Then, in a second stage, two concentric spheres of liquid water separated by $10 \mu \mathrm{m}$ were considered. The inner sphere radius varies between $15 \mu \mathrm{m}$ and $250 \mu \mathrm{m}$, representing colloids, a component of the thyroid tissue, whereas the surrounding $10-\mu \mathrm{m}$ thick region represents follicular cells, as found in the literature [16]. The electron emission is isotropic and distributed uniformly in the inner sphere. Emitted electrons are shot according to the emission spectrum of five isotopes of iodine (from 131 to 135), presented in [16], [32]. For each spectrum, $5 \times 10^{4}$ incident electrons are considered, each of them corresponding to one of the following emission processes: $\beta$-emission, Auger emission or internal conversion. The emission spectra of these five isotopes for $5 \times 10^{4}$ electrons are presented in Fig. 1, for long-life (131) and short-life $\left({ }^{132}\left|,{ }^{133}\right|,{ }^{134} \mid\right.$, and ${ }^{135} \mid$ ) iodine isotopes. On this figure, processes are also distinguished. The absorbed dose is reckoned both in the inner sphere and in the surrounding region, located between the two concentric spheres. 

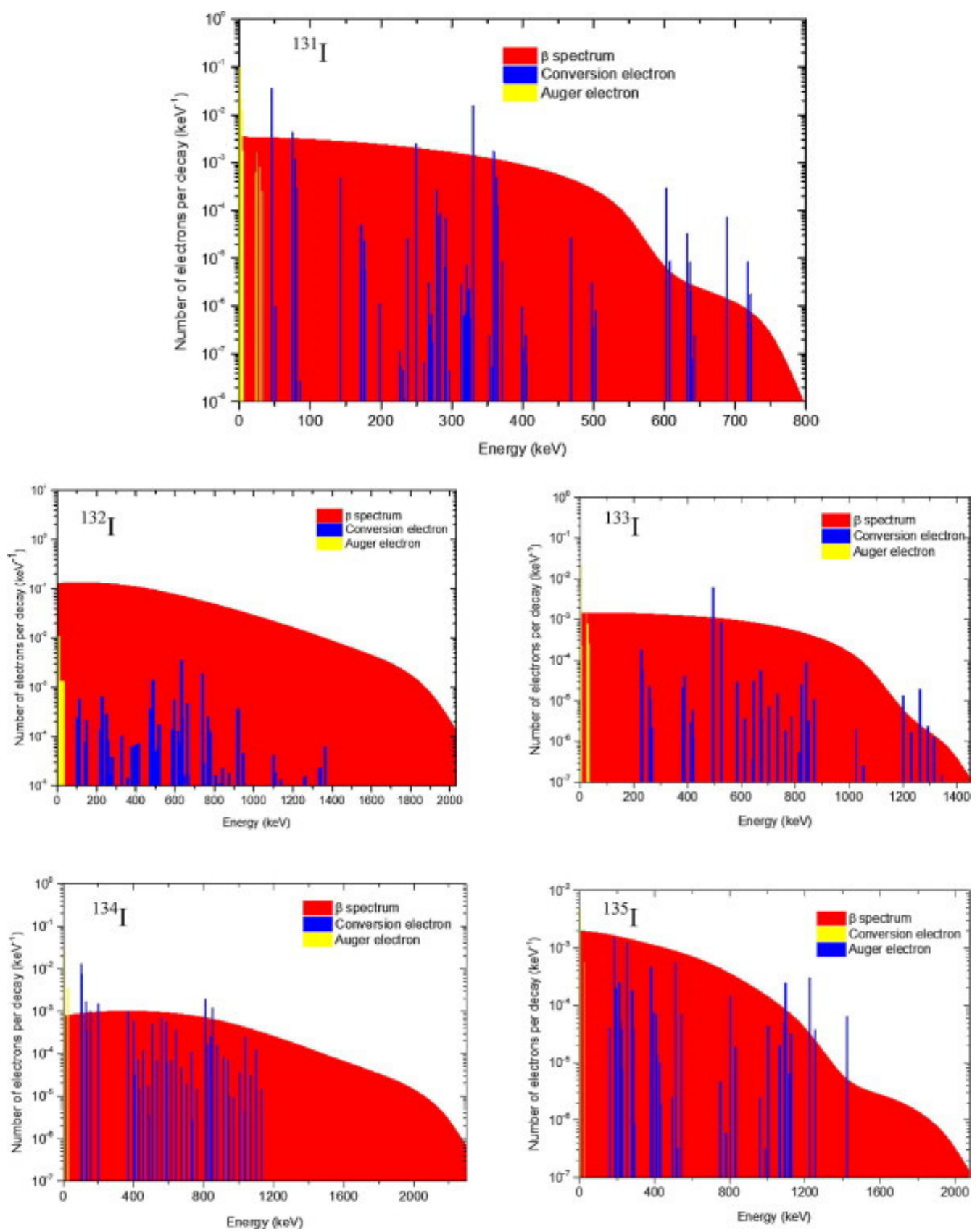

Fig. 1. Emission spectra of ${ }^{131}$ ( (long-life isotope), ${ }^{132}$ |, ${ }^{133 \mid}$, ${ }^{134}$ | and ${ }^{135}$ ( (short-life isotopes). Beta continuum, Auger electrons and internal conversion electrons are distinguished.

\subsection{Data processing}

The S-value simulation code generates ROOT output files, which are later processed using the ROOT data analysis framework [33]. The $S$-values are obtained at this stage, by computing the absorbed dose per disintegration, i.e. by dividing the absorbed dose (whether the total, or the one from each process) by $5 \times 10^{4}$.

In the case of iodine isotopes, we compute both the $S$-value from the total absorbed dose and the contribution of each process. The final values are obtained after a correction, taking into account the fact that the emission spectra also contain X-ray photons. Therefore, it is necessary to use the following correction factor: 


$$
S_{\text {corr }}=S_{\text {init }} \times \frac{\bar{E}_{a c t}}{\bar{E}}
$$

where $S_{\text {init }}$ is the absorbed dose converted to a single disintegration (i.e. divided by $5 \times 10^{4}$ ), and $S_{\text {corr }}$ is the final corrected value, $\bar{E}_{\text {act }}$ is the actual average released disintegration energy (considering all physical processes, including X-ray photons), and $\bar{E}$ is the average released disintegration energy obtained from the spectrum used in the simulation code (which includes $5 \times 10^{4}$ electrons only, including the three emission processes). The values of those average energies as well as the correction factor $\frac{\bar{E}_{a c t}}{\bar{E}}$ calculated as described in reference [16] are presented in Table 2 for each iodine isotope.

Table 2. For each iodine radioisotope, this table presents the values of $\bar{E}_{\text {act }}$ the actual average released disintegration energy (considering all physical processes, including X-ray photons), and $\bar{E}$, which is the average released disintegration energy obtained from the spectrum used in the simulation code (which includes $5 \times 10^{4}$ electrons only, including the three emission processes). The correction factor is given by the ratio $\frac{\bar{E}_{\text {act }}}{\bar{E}}$

\begin{tabular}{|l|l|l|l|}
\hline Isotope & $\bar{E}_{\text {act }}(\mathrm{keV})$ & $\bar{E}(\mathrm{keV})$ & Correction factor $\frac{\bar{E}_{\text {act }}}{\bar{E}}$ \\
\hline${ }_{131} \mid$ & 184.9 & 155.4 & 1.19 \\
\hline${ }_{132 \mid}$ & 498.5 & 461.9 & 1.08 \\
\hline${ }_{133 \mid}$ & 416.1 & 390.5 & 1.07 \\
\hline${ }_{134 \mid}^{135 \mid}$ & 630.5 & 556.0 & 1.13 \\
\hline
\end{tabular}

\subsection{Comparison method}

For monoenergetic electrons, Geant4-DNA results for $S$-values are compared to simulation results computed with three other Monte Carlo codes, obtained either from the literature (for the in-house code MC4V developed by Emfietzoglou et al.[31]) or from dedicated simulations performed at the LAPLACE laboratory, in Toulouse (for the CPA100 code and for the PENELOPE 2011 code). Regarding isotopes of iodine, our results are compared with those obtained from EGSnrc, PENELOPE 2006, MCNP and CELLDOSE and performed specifically for this work. PENELOPE 2006 results have been obtained at the CEA-LIST laboratory in Gifsur-Yvette, France. The use of two different versions of PENELOPE does not introduce any bias in the results because the physics remains unchanged between version 2006 and version 2011.

In both cases (monoenergetic electrons and isotopes of iodine), the comparison has been carried out by using Kolmogorov-Smirnov (KS) statistical tests. In the monoenergetic case, the statistical KS test was applied only to monotonic sections of the curve giving the dependence of the $S$-value as a function of the sphere radius. On the contrary, in the radionuclide case, the KS test was applied to the whole monotonous curves. In addition to the $\mathrm{KS}$ test, the relative difference (as a percentage) between Geant4-DNA and each code has been calculated as follows: 


$$
R D=\left(\frac{S_{\text {value }}(\text { Code })}{S_{\text {value }}(\text { GEANT4 }-D N A)}-1\right) \times 100 \%
$$

Furthermore, the results obtained with $131 \mathrm{I}$ for the total S-value are compared to the data from Bardiès and Chatal [27].

\section{Results and discussion}

\subsection{Monoenergetic electrons}

The results obtained for monoenergetic electrons with Geant4-DNA, CPA100, PENELOPE and MC4 V [34] are presented in Fig. 2. The simulations have been carried out in liquid water spheres with radii of $10,50,100$ and $1000 \mathrm{~nm}$, and for incident energies ranging from $100 \mathrm{eV}$ to $20 \mathrm{keV}$. We found that the Geant4-DNA simulations are statistically compatible with the other simulations ( $p$-value $>0.05)$. The maximum disagreement $(D)$ reaches 0.5 , and is obtained between Geant4-DNA and the three other codes for a radius of $10 \mathrm{~nm}$ (the lowest sphere radius).
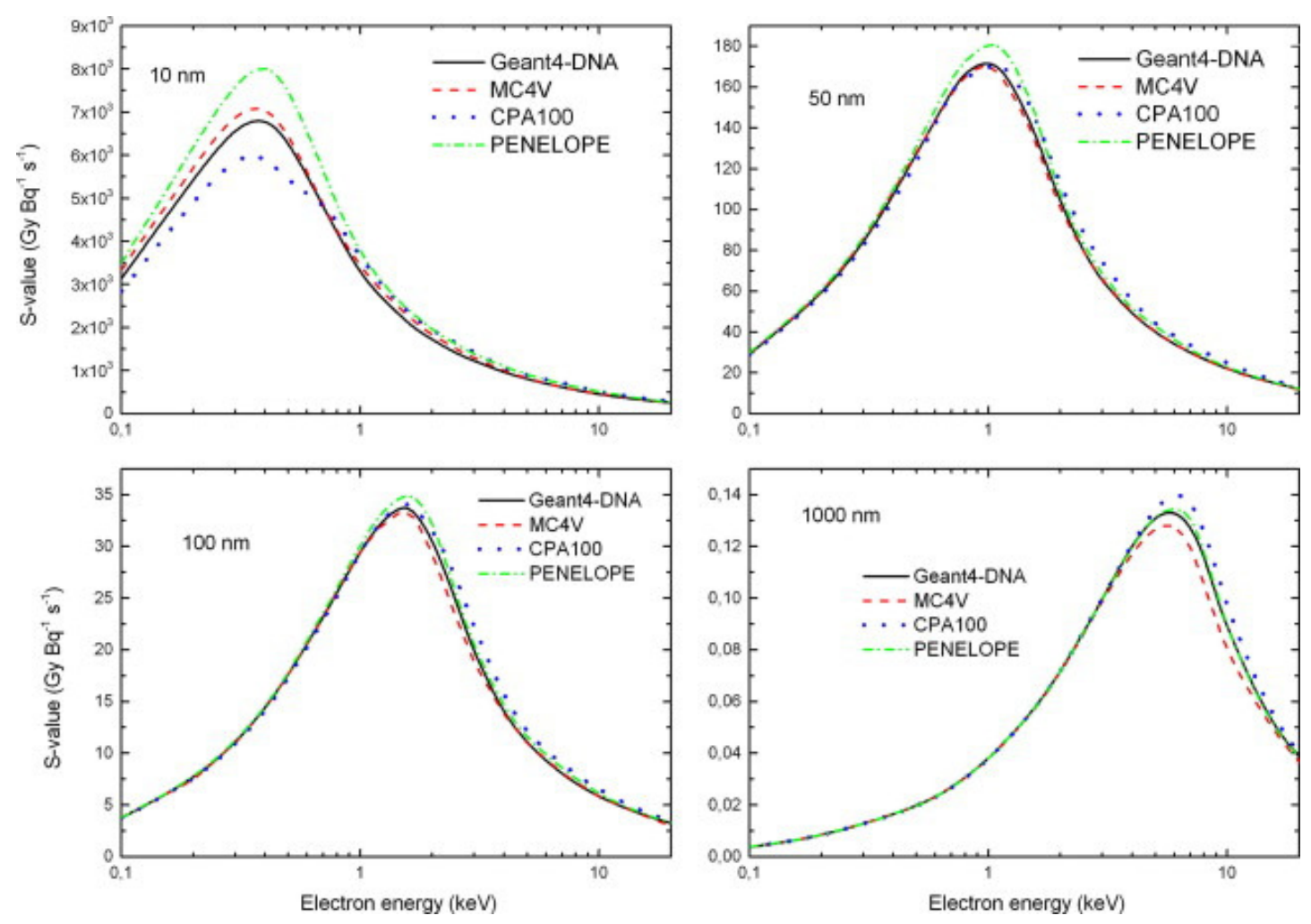

Fig. 2. Variations of $S$-values for monoenergetic electrons emitted in a liquid water sphere for the following radii: 10, 50, 100 and $1000 \mathrm{~nm}$. Emfietzoglou et al. [34] in-house code MC4 V, CPA100 and PENELOPE are compared to Geant4-DNA.

The corresponding relative differences between Geant4-DNA and these codes lie between $-15 \%$ and $+20 \%$ for all codes and energies. The best agreement with Geant4-DNA is obtained for the results of MC4V [34] (e.g. the relative differences lie between $-3.6 \%$ and $+1.7 \%$ for a sphere radius of $50 \mathrm{~nm}$ ), except for a radius of $1000 \mathrm{~nm}$, where PENELOPE shows a better agreement with Geant4-DNA (between $-1.2 \%$ and $+3.5 \%$ ). 


\subsection{Isotopes of iodine}

\subsubsection{Colloid case}

As an illustration, S-values obtained with Geant4-DNA for ${ }^{131}$ as a function of colloid sphere radius, varying from $10 \mu \mathrm{m}$ to $250 \mu \mathrm{m}$, are shown in Fig. 3. These results are compared to EGSnrc, CELLDOSE, MCNP and PENELOPE Monte Carlo codes and to Bardiès and Chatal data [27]. In this figure, $S$-values have been calculated for all processes (top-left plot), $\beta^{-}$emission (top-right plot), internal conversion (bottom-left plot) and Auger emission (bottom-right plot).

\section{Colloid}
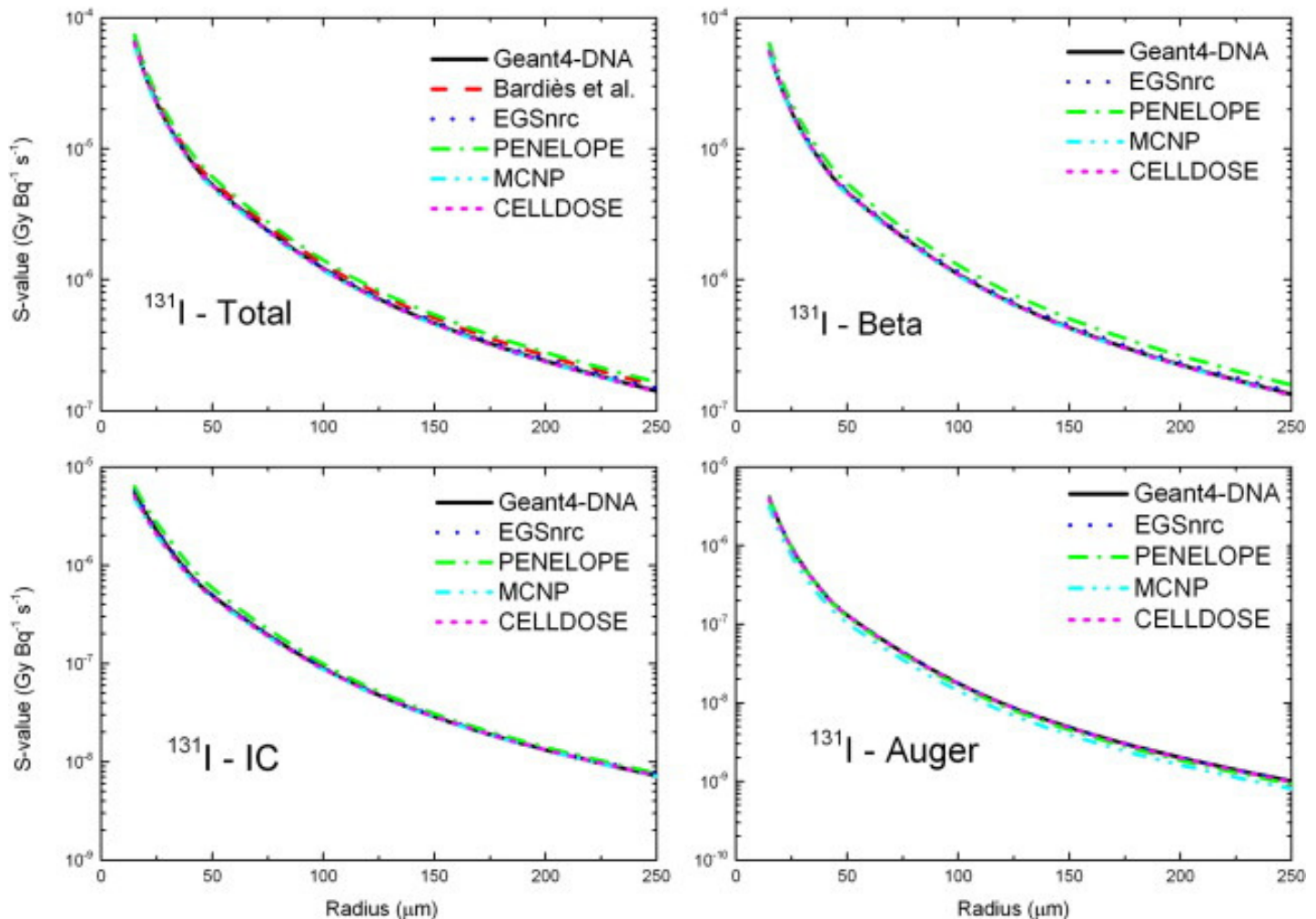

Fig. 3. Dependence of $S$-values obtained for ${ }^{131} \mid$ as a function of colloid sphere radius, varying from $10 \mu \mathrm{m}$ to $250 \mu \mathrm{m}$. Geant4-DNA results are compared to EGSnrc, CELLDOSE, MCNP and PENELOPE Monte Carlo codes and to Bardiès and Chatal data [27]. S-values have been calculated for all processes (top-left), beta emission (top-right), internal conversion (bottomleft) and Auger emission (bottom-right).

If we consider all codes, isotopes and processes, the relative difference (not shown) varies between $-22.50 \%$ and $+75 \%$ in some extreme cases. In most of the cases however, the difference range is much smaller, as for CELLDOSE for ${ }^{131}$ (between $-8 \%$ and $+0.75 \%$ ). Globally, Geant4-DNA matches CELLDOSE to the greatest extent for the simulations in the colloid. In some cases, however, EGSnrc (e.g. below $6.5 \%$ for ${ }^{133}$, not graphically shown) or MCNP (e.g. mostly below $10 \%$ for ${ }^{134}$ I) are closer. We can notice, especially for ${ }^{133}$, a strong similarity in the variations for EGSnrc, MCNP and CELLDOSE. The data from Bardiès and Chatal [27] for ${ }^{131}$ I in the colloid for the total $S$-value are from $7 \%$ up to $10 \%$ larger than those of Geant4-DNA. 


\subsubsection{Follicular cells case}

For example, S-values obtained with Geant4-DNA for ${ }^{134}$ | for follicular cells are shown in Fig. 4. These results are compared to EGSnrc, CELLDOSE, MCNP and PENELOPE Monte Carlo codes. In this figure, $S$-values have been calculated for all processes (top-left plot), beta emission (top-right plot), internal conversion (bottom-left plot) and Auger emission (bottomright plot).

\section{Follicular}
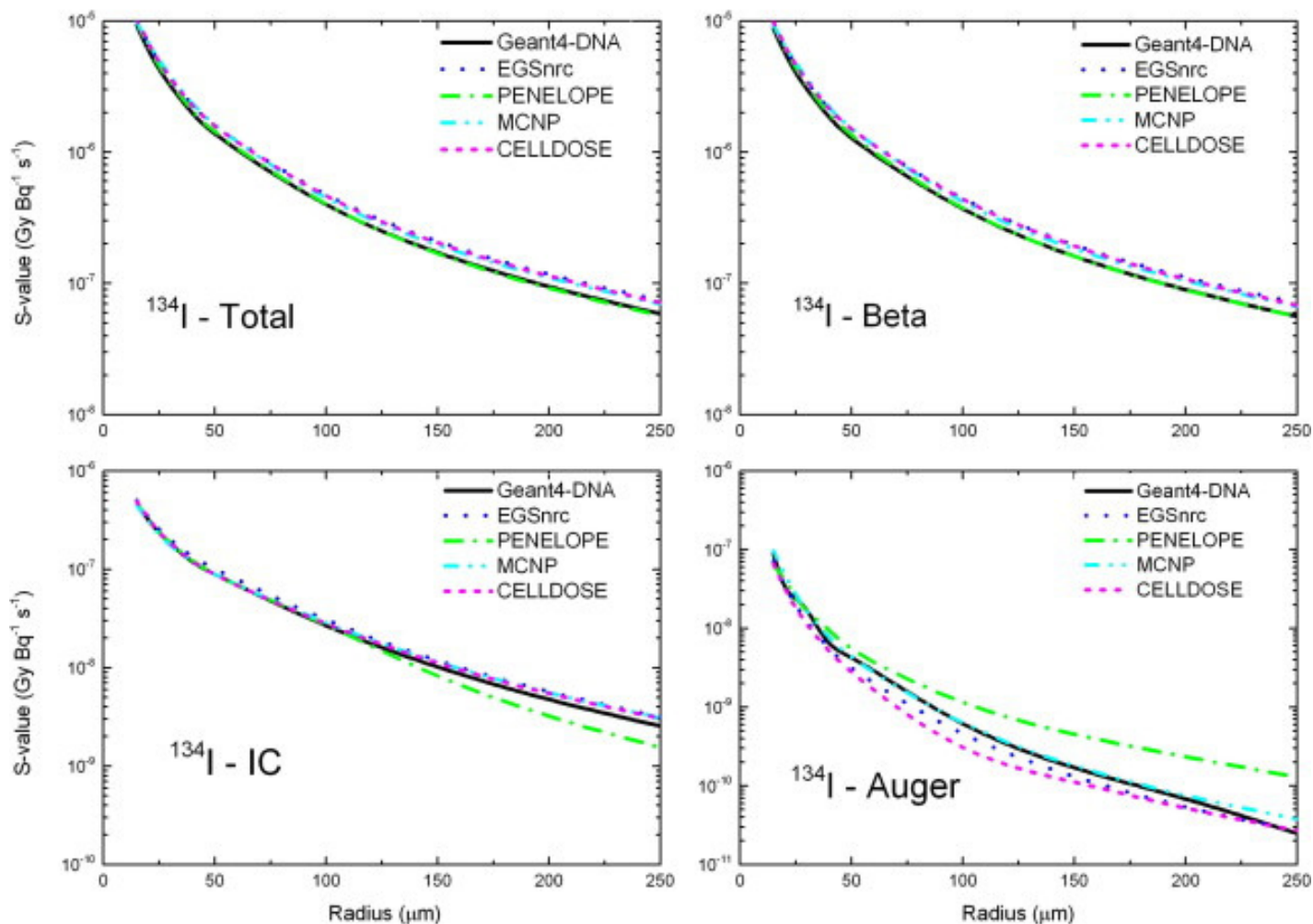

Fig. 4. Dependence of $S$-values obtained for ${ }^{134} \mathrm{I}$ as a function of follicular cells for radius varying from $10 \mu \mathrm{m}$ to $250 \mu \mathrm{m}$. Geant4-DNA results are compared to EGSnrc, CELLDOSE, MCNP and PENELOPE Monte Carlo codes. S-values have been calculated for all processes (top-left), beta emission (top-right), internal conversion (bottom-left) and Auger emission (bottom-right).

There is general good agreement between Geant4-DNA and all codes, which show the Svalue global dependence as a function of colloid radius. However, for Auger emission, the PENELOPE results deviate from the other codes, becoming higher for large radii. We attribute this behaviour which is not observed with the other codes to a lack of statistics on Auger electrons.

In any case, for both cases (colloid and follicular cells), regarding the KS statistical tests, it was found that all $p$-values were larger than 0.05 , confirming the overall statistical agreement with Geant4-DNA results. 


\section{Conclusions}

The results presented in this work are part of the verification efforts to evaluate the accuracy of Geant4-DNA physics models for the transport of electrons in liquid water. We have determined $S$-values for monoenergetic electrons and spectra from the decay of iodine radioisotopes (131|, 132|, 133|, 134|, 135|), obtained respectively in nanometer size spheres and in two geometrical descriptions of thyroid components. The results obtained with Geant4-DNA have been compared to results obtained from other Monte Carlo codes and from the literature. Kolmogorov-Smirnov tests have shown that all of them are statistically compatible with Geant4-DNA. For the monoenergetic electron spectra, the best agreement with Geant4-DNA is obtained for MC4V [34], while in the case of iodine radioisotopes calculated in the colloid, CELLDOSE results show the best agreement with the Geant4-DNA determinations. The results obtained for the follicular cell model by use of the codes in question show a general good agreement, except for Auger electrons simulated with PENELOPE at large radii. The statistical compatibility remains however verified.

Apart from specific transport parameters which the user can tune for dedicated applications, the various Monte Carlo codes used in this work adopt different theoretical and/or semiempirical models (see [15], [21], [22], [23], [35], [25]) in order to simulate electron physical interactions (such as ionization, electronic excitation and elastic scattering) in liquid water. Simulated $S$-values are obtained directly from the scoring of elementary energy deposition events by electrons in small size targets of liquid water. As a consequence, variations observed between the codes are mainly caused by differences in stopping powers (see for example the discussion in Section 3.1 of [36]). In a further work, it would be interesting to accurately quantify these differences in stopping powers in order to try to identify which models could be further improved.

\section{Acknowledgements}

The Geant4-DNA project receives funding from the European Space Agency under contract number AO7146-4000107387/12/NL/AK (2013-2016). It is also supported by the INSERM PhysiCancer "MICRONAUTE" project (2013-2014). Thibault André was supported by a dedicated INSERM Cancéropôle Grand Sud Ouest funding for this work, under contract number 2012140602. Filippo Morini is post-doctoral fellow from the FWO at Hasselt University. M.A. Bernal thanks the FAPESP foundation in Brazil for financing his research activities through the 2011/51594-2 project.

\section{References}

[1] Brachytherapy: Applications and Techniques, Lippincott Williams \& Wilkins, 2007.

[2] J.P. Vuillez. Traitements à visée palliative ou curative: la radiothérapie vectorisée des tumeurs endocrines. Médecine Nucléaire, 31 (2007), pp. 506-513

[3] A.I. Kassis, S.J. Adelstein. Radiobiologic principles in radionuclide therapy. J. Nucl. Med., 46 (2005), pp. 4S-12S

[4] J. Barbet, F. Kraber-Bodéré, A. Faivre-Chauvet, J.-F. Gestin, M. Bardiès, L. Campion, J.-F. Chatal. La radio-immunothérapie préciblée des tumeurs solides: une démarche pluridisciplinaire. Médecine Nucléaire, 31 (2007), pp. 498-505

[5] F. Buchegger, F. Perillo-Adamer, Y. Dupertuis, A. Bischof Delaloye. Auger radiation targeted into DNA: a therapy perspective. Eur. J. Nucl. Med. Mol. Imaging, 33 (2006), pp. $1352-1363$ 
[6] F. Giammarile, F. Borson-Chazot. Traitement des tumeurs neuroendocrines par la mIBG et les peptides radiomarqués. Pathol. Biol. (Paris), 54 (2006), pp. 130-136

[7] L. Bodei, F. Giammarile. Traitement des tumeurs neuro-endocrines par les peptides radiomarqués. Médecine Nucléaire, 33 (2009), pp. 142-147

[8] S. Brillouet, S. Dorbes, S. Silvente-Poirot, B. Mestre-Voegtlé, C. Picard, M. Poirot, F. Courbon. Ciblage peptidique en oncologie nucléaire: intérêt de la modélisation moléculaire. Médecine Nucléaire, 34 (2010), pp. 289-294

[9] W.S. Snyder, M.R. Ford, G.G. Warner, S.B. Watson. S - absorbed dose per unit cumulated activity for selected radionuclides and organs NM/MIRD pamphlet No. 11, in, Oak Ridge National Laboratory, 1975.

[10] W.E. Bolch, L.G. Bouchet, J.S. Robertson, B.W. Wessels, J.A. Siegel, R.W. Howell, A.K.Erdi, B. Aydogan, S. Costes, E.E. Watson, A.B. Brill, N.D. Charkes, D.R. Fisher, M.T. Hays, S.R. Thomas. MIRD pamphlet No. 17: the dosimetry of nonuniform activity distributions-radionuclide $S$ values at the voxel level. Medical internal radiation dose committee. J. Nucl. Med., 40 (1999), pp. 11S-36S

[11] W.E. Bolch, K.F. Eckerman, G. Sgouros, S.R. Thomas. MIRD pamphlet No. 21: a generalized schema for radiopharmaceutical dosimetry-standardization of nomenclature. J. Nucl. Med., 50 (2009), pp. 477-484

[12] C. Champion, A. L'Hoir, M.F. Politis, P.D. Fainstein, R.D. Rivarola, A. Chetioui. A Monte Carlo code for the simulation of heavy-ion tracks in water. Radiat. Res., 163 (2005), pp. 222-231

[13] C. Champion, C. Le Loirec. Positron follow-up in liquid water: II. Spatial and energetic study for the most important radioisotopes used in PET. Phys. Med. Biol., 52 (2007), pp. 6605-6625

[14] C. ChampionTheoretical cross sections for electron collisions in water: structure of electron tracks. Phys. Med. Biol., 48 (2003), pp. 2147-2168

[15] S. Incerti, A. Ivanchenko, M. Karamitros, A. Mantero, P. Moretto, H.N. Tran, B.Mascialino, C. Champion, V.N. Ivanchenko, M.A. Bernal, Z. Francis, C. Villagrasa, G.Baldacchino, P. Guèye, R. Capra, P. Nieminen, C. Zacharatou. Comparison of GEANT4 very low energy cross section models with experimental data in water. Med. Phys., 37 (2010), pp. 46924708

[16] C. Champion, P. Zanotti-Fregonara, E. Hindié. CELLDOSE: a Monte Carlo code to assess electron dose distribution- $S$ Values for 131 in spheres of various sizes. J. Nucl. Med., 49 (2008), pp. 151-157

[17] S. Incerti, G. Baldacchino, M. Bernal, R. Capra, C. Champion, Z. Francis, P. Guèye, A.Mantero, B. Mascialino, P. Moretto, P. Nieminen, C. Villagrasa, C. Zacharatou. The Geant4-DNA project. Int. J. Model. Simul. Sci. Comput., 01 (2010), pp. 157-178

[18] Z. Francis, S. Incerti, R. Capra, B. Mascialino, G. Montarou, V. Stepan, C. Villagrasa. Molecular scale track structure simulations in liquid water using the Geant4-DNA Monte-Carlo processes. Appl. Radiat. Isot., 69 (2011), pp. 220-226

[19] S. Agostinelli, J. Allison, K. Amako, J. Apostolakis, H. Araujo, P. Arce, M. Asai, D. Axen, S.Banerjee, G. Barrand, F. Behner, L. Bellagamba, J. Boudreau, L. Broglia, A. Brunengo, H.Burkhardt, S. Chauvie, J. Chuma, R. Chytracek, G. Cooperman, G. Cosmo, P.Degtyarenko, A. Dell'Acqua, G. Depaola, D. Dietrich, R. Enami, A. Feliciello, C.Ferguson, H. Fesefeldt, G. Folger, F. Foppiano, A. Forti, S. Garelli, S. Giani, R.Giannitrapani, D. Gibin, J.J. Gómez 
Cadenas, I. González, G. Gracia Abril, G. Greeniaus, W. Greiner, V. Grichine, A. Grossheim, S. Guatelli, P. Gumplinger, R. Hamatsu, K.Hashimoto, H. Hasui, A. Heikkinen, A. Howard, V. Ivanchenko, A. Johnson, F.W. Jones, J. Kallenbach, N. Kanaya, M. Kawabata, Y. Kawabata, M. Kawaguti, S. Kelner, P. Kent, A.Kimura, T. Kodama, R. Kokoulin, M. Kossov, H. Kurashige, E. Lamanna, T. Lampén, V.Lara, V. Lefebure, F. Lei, M. Liendl, W. Lockman, F. Longo, S. Magni, M. Maire, E.Medernach, K. Minamimoto, P. Mora de Freitas, Y. Morita, K. Murakami, M. Nagamatu, R. Nartallo, P. Nieminen, T. Nishimura, K. Ohtsubo, M. Okamura, S. O'Neale, Y. Oohata, K. Paech, J. Perl, A. Pfeiffer, M.G. Pia, F. Ranjard, A. Rybin, S. Sadilov, E. Di Salvo, G.Santin, T. Sasaki, N. Savvas, Y. Sawada, S. Scherer, S. Sei, V. Sirotenko, D. Smith, N.Starkov, H. Stoecker, J. Sulkimo, M. Takahata, S. Tanaka, E. Tcherniaev, E. Safai Tehrani, M. Tropeano, P. Truscott, H. Uno, L. Urban, P. Urban, M. Verderi, A. Walkden, W..Wander, H. Weber, J.P. Wellisch, T. Wenaus, D.C. Williams, D. Wright, T. Yamada, H.Yoshida, D. Zschiesche. G4-a simulation toolkit. Nucl. Instr. Meth. Phys. Res. Sect. A, 506 (2003), pp. 250-303

[20] J. Allison, K. Amako, J. Apostolakis, H. Araujo, P.A. Dubois, M. Asai, G. Barrand, R.Capra, S. Chauvie, R. Chytracek, G.A.P. Cirrone, G. Cooperman, G. Cosmo, G. Cuttone, G.G. Daquino, M. Donszelmann, M. Dressel, G. Folger, F. Foppiano, J. Generowicz, V.Grichi ne, S. Guatelli, P. Gumplinger, A. Heikkinen, I. Hrivnacova, A. Howard, S. Incerti, V. Ivanche nko, T. Johnson, F. Jones, T. Koi, R. Kokoulin, M. Kossov, H. Kurashige, V.Lara, S. Larsson, F. Lei, O. Link, F. Longo, M. Maire, A. Mantero, B. Mascialino, I.McLaren, P.M. Lorenzo, K. Minamimoto, K. Murakami, P. Nieminen, L. Pandola, S.Parlati, L. Peralta, J. Perl, A. Pfeiffer, M.G. Pia, A. Ribon, P. Rodrigues, G. Russo, S.Sadilov, G. Santin, T. Sasaki, D. Smith, N. St arkov, S. Tanaka, E. Tcherniaev, B. Tome, A.Trindade, P. Truscott, L. Urban, M. Verderi, A. Walkden, J.P. Wellisch, D.C. Williams, D.Wright, H. Yoshida. Geant4 developments and applications. Nucl. Sci., 53 (2006), pp. 270-278

[21] M. Terrissol, A. Beaudré. Simulation of space and time evolution of radiolytic species induced by electrons in water. Radiat. Prot. Dosimetry, 31 (1990), pp. 175-177

[22] I. Kawrakow. Accurate condensed history Monte Carlo simulation of electron transport. I. [small-caps EGS]nrc, the new [small-caps EGS4] version. Med. Phys., 27 (2000), pp. 485-498

[23] C. Champion, C. Le Loirec, B. Stosic. EPOTRAN: a full-differential Monte Carlo code for electron and positron transport in liquid and gaseous water. Int. J. Radiat. Biol., 88 (2012), p. 54

[24] J.T. Goorley, M.R. James, T.E. Booth, F.B. Brown, J.S. Bull, L.J. Cox, J.W. Durkee, Jr., J.S. Elson, M.L. Fensin, R.A. Forster, III, J.S. Hendricks, H.G. Hughes, III, R.C. Johns, B.C. Kiedrowski, R.L. Martz, S.G. Mashnik, G.W. McKinney, D.B. Pelowitz, R.E. Prael, J.E. Sweezy, L.S. Waters, T. Wilcox, A.J. Zukaitis, Initial MCNP6 Release Overview-MCNP6 version 1.0, in, 2013, pp. Medium: ED.

[25] J. Baró, J. Sempau, J.M. Fernández-Varea, F. Salvat. PENELOPE: an algorithm for Monte Carlo simulation of the penetration and energy loss of electrons and positrons in matter. Nucl. Instr. Meth. Phys. Res. Sect. B, 100 (1995), pp. 31-46

[26] Y. Perrot, Evaluation de la dose déposée par des faisceaux d'électrons en radiothérapie dans des fantômes voxelisés en utilisant la plateforme de simulation Monte Carlo GATE fondée sur GEANT4 dans un environnement de grille, in Université Blaise Pascal - ClermontFerrand II, 2012. 
[27] M. Bardies, J.F. Chatal. Absorbed doses for internal radiotherapy from 22 betaemitting radionuclides: beta dosimetry of small spheres. Phys. Med. Biol., 39 (1994), pp. $961-981$

[28] W.B. Li, W. Friedland, E. Pomplun, P. Jacob, H.G. Paretzke, M. Lassmann, C. Reiners. Track structures and dose distributions from decays of $131 \mathrm{I}$ and $125 \mathrm{I}$ in and around water spheres simulating micrometastases of differentiated thyroid cancer. Radiat. Res., 156 (2001), pp. 419-429

[29] S.M. Goddu, R.W. Howell, D.V. Rao. Cellular dosimetry: absorbed fractions for monoenergetic electron and alpha particle sources and $S$-values for radionuclides uniformly distributed in different cell compartments. J. Nucl. Med., 35 (1994), pp. 303-316

[30] S.M. Goddu, D.V. Rao, R.W. Howell. Multicellular dosimetry for micrometastases: dependence of self-dose versus cross-dose to cell nuclei on type and energy of radiation and subcellular distribution of radionuclides. J. Nucl. Med., 35 (1994), pp. 521 530

[31] D. Emfietzoglou, K. Kostarelos, P. Hadjidoukas, C. Bousis, A. Fotopoulos, A. Pathak, H.Nikjoo. Subcellular S-factors for low-energy electrons: a comparison of Monte Carlo simulations and continuous-slowing-down calculations. Int. J. Radiat. Biol., 84 (2008), pp. 1034-1044

[32] C. Champion, M. Elbast, T.-D. Wu, N. Colas-Linhart. Thyroid cell irradiation by radioiodines: a new Monte Carlo electron track-structure code. Braz. Arch. Biol. Technol., 50 (2007), pp. 135-144

[33] R. Brun, F. Rademakers. ROOT - an object oriented data analysis framework. Nucl. Instr. Meth. Phys. Res. Sect. A, 389 (1997), pp. 81-86

[34] D. Emfietzoglou, C. Bousis, C. Hindorf, A. Fotopoulos, A. Pathak, K. Kostarelos. A Monte Carlo study of energy deposition at the sub-cellular level for application to targeted radionuclide therapy with low-energy electron emitters. Nucl. Instr. Meth. Phys. Res. Sect. B, 256 (2007), pp. 547-553

[35] R.A. Forster, L.J. Cox, R.F. Barrett, T.E. Booth, J.F. Briesmeister, F.B. Brown, J.S. Bull, G.C. Geisler, J.T. Goorley, R.D. Mosteller, S.E. Post, R.E. Prael, E.C. Selcow, A. Sood. MCNP TM Version 5. Nucl. Instr. Meth. Phys. Res. Sect. B, 213 (2004), pp. 82-86

[36] S. Incerti, C. Champion, H.N. Tran, M. Karamitros, M. Bernal, Z. Francis, V. Ivanchenko, A. Mantero. Energy deposition in small-scale targets of liquid water using the very low energy electromagnetic physics processes of the Geant4 toolkit. Nucl. Instr. Meth. Phys. Res. Sect. B, 306 (2013), pp. 158-164 